\title{
THE CLINICAL CONUNDRUM OF A NUMB CHIN
}

N. Ghazali, S.M. Ismail, Z.A. Abdul Rahman. The Clinical Conundrum of A Numb Chin. Annal Dent Univ Malaya 2001; 8: 67-74.

\begin{abstract}
Mental nerve neuropathy is an important presenting complaint that may be encountered by dental surgeons in their daily practise. There are various pathological processes that could bring about this symptom, ranging from simple dental cause to vague, life threatening diseases. We present three cases of mental paraesthesia of different aetiologies. A literature review on mental nerve neuropathy related to malignancies and infection is discussed. The importance of a thorough chair side history taking, clinical examination and relevant investigations are emphasised in a suggested clinical approach to obtaining the diagnosis of a numb chin.
\end{abstract}

Key words: Mental nerve - Numb Chin Syndrome Paraesthesia - Anaesthesia - Neuropathy - Infection Metastatic tumor- Differential diagnosis

\section{INTRODUCTION}

Numbness and tingling sensation is a condition very frequently encountered by the dentist in the dental practice. Paraesthesia is defined as a spontaneously occurring abnormal touch sensation like burning and tingling while anaesthesia refers to the complete loss of sensation in an area of nerve distribution. These are possible presentations resulting from an alteration to the normal function of a nerve termed dysesthesia, which may be caused by diverse pathological processes (Figure 1) affecting the intra-cranial or extra-cranial course of the nerve or as a part of a systemic disorder.

We present three patients with mental nerve dysfunction of different aetiologies with an emphasis on highlighting the importance of a thorough clinical history, physical examination and appropriate investigations in unravelling their causes.

\section{CASE REPORTS}

\section{Case 1}

\section{History}

A 50-year old Mailay lady was referred to the Department of Oral \& Maxillofacial Facial Surgery by her general dental practitioner (GDP) with a week history of mental nerve paraesthesia associated with right facial swelling. The tingling sensation on the lower lip began after the extraction of a carious right first mandibular molar, which was performed without complication under local anaesthesia. Two days

\section{Article of Interest}

N. Ghazali1 ${ }^{1}$, S.M. Ismail ${ }^{2}$ and

Z.A. Abdul Rahman²

'Department of Oral \& Maxillofacial Surgery, Faculty of Dentistry,

National University of Malaysia (UKM), Jalan Raja Muda Abdul Aziz, 50300 Kuala Lumpur

${ }^{2}$ Department of Oral \& Maxillofacial Surgery, Faculty of Dentistry, University of Malaya, 50603 Kuala Lumpur, MALAYSIA.

Corresponding author - Dr Naseem Ghazali

following the extraction, the patient developed a painful right facial swelling associated with 'pins and needles' in the right half of the lip and chin. She returned to the GDP who observed an empty socket and sequestrum at the extraction site. A diagnosis of dry socket was reached and the socket was irrigated and dressed under local anaesthesia. A 5-day course of Ampicillin and Metronidazole was prescribed but failed to bring about improvement. She denied other neurological problems and systems review did not reveal any other abnormalities. Her medical history was unremarkable except for a history of gestational diabetes mellitus 15 years ago, however, she was not diabetic at presentation.

\section{Clinical examination}

Extra-oral examination revealed an ill-defined, diffuse swelling involving the right body of mandible, submandibular lymphadenopathy and paraesthesia over the distribution of the inferior alveolar nerve, especially on the mental region. The post-extraction site was inflammed and the socket was filled with debris. Fragments of sequestered bone were seen protruding from beneath the inflamed gums.

\section{Investigation}

Two initial radiographic films were taken. The dental orthopantomogram (OPG) film showed multiple, irregular, lobulated, radiopaque masses that occurred mainly in the premolar-molar region, bilaterally, in the mandible and maxilla. The masses in the mandible were seen near the alveolar crest while those in the maxilla were located beyond the apices of maxillary teeth. Generally, the masses had well-defined margins with the exception of the post-extraction site, which had a cuff of reduced radio-density described as 'moth-eaten' appearance. An occlusal view of the mandible showed a bony expansion involving the right body of mandible. Widespread bony lesions were ruled out after further radiological investigations.

A pus swab was obtained from extraction socket for culture and sensitivity. This revealed moderate growth 


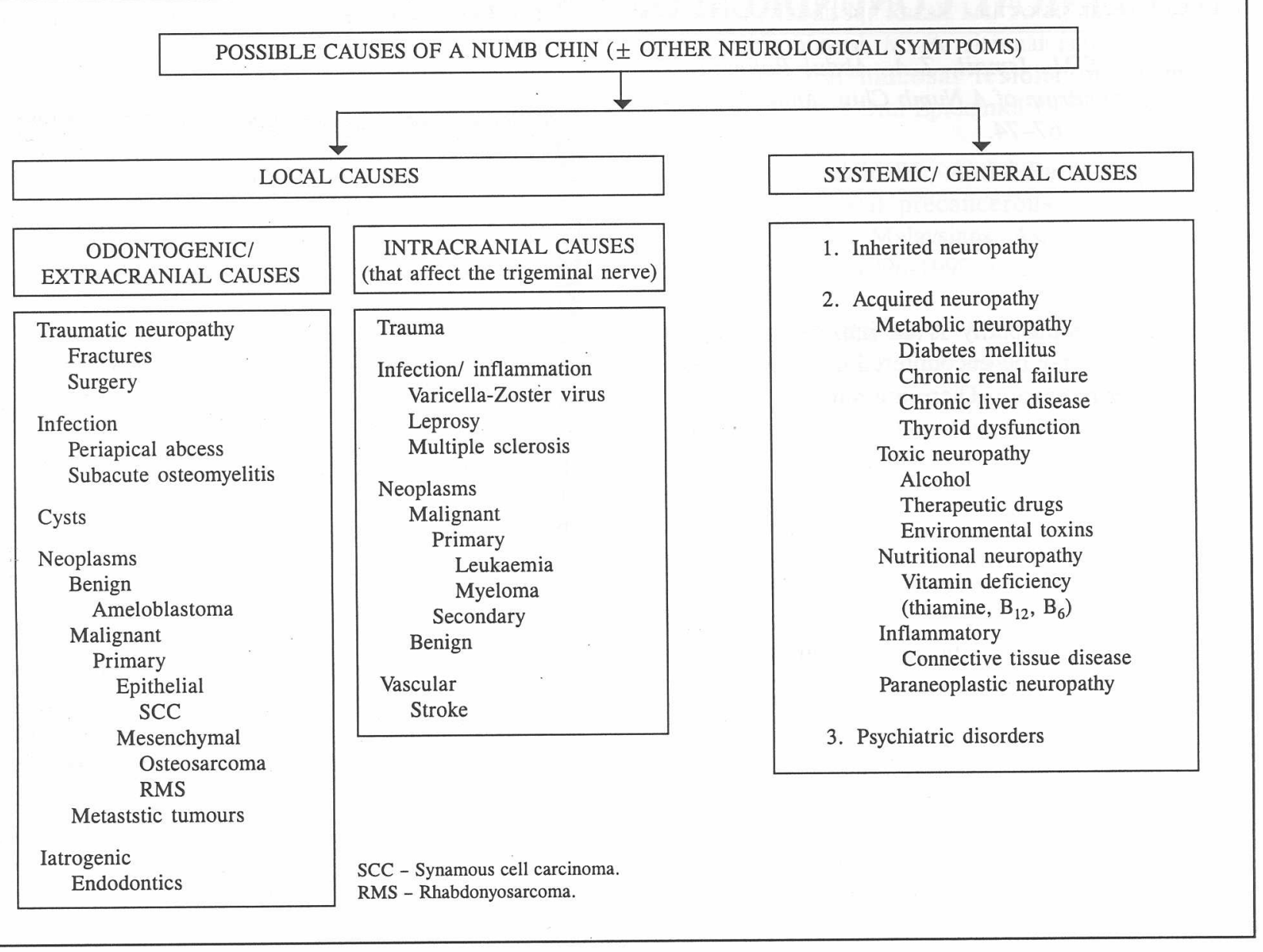

Figure 1: Causes of mental paraesthesia.

of coagulase-positive methicillin-sensitive Staphylococus aureus. Fasting blood glucose and serum alkaline phosphatase levels were within normal limits.

\section{Diagnosis}

In keeping with the overall clinical picture, a provisional diagnosis of subacute osteomyelitis on an existing florid cemento-osseous dysplasia was reached. The patient was subjected to debridement under general anaesthesia to allow for adequate sequestrectomy and removal of the surrounding mass of dense calcified material that was sent for histopathological examination, the result of which was in agreement with the provisional diagnosis. The bony defect was packed with ribbon gauze soaked in Whitehead's varnish and the patient was prescribed flucloxacillin and metronidazole for a week. The wound healed uneventfully and normal mental sensation was restored once the infection resolved.

\section{Case 2}

\section{History}

A 9-year old Chinese boy was referred to the Department of Oral \& Maxillofacial Surgery with a 1 month history of loss of sensation on the right side of his chin, lower lip and right half of tongue. This was associated with altered speech, siallorhoea in the presence of intact taste sensation. Within the same period, a progressive swelling developed on the right side of his face. Two months prior to this, he complained of severe persistent headache with no obvious aggravating or relieving factors. Headache was not associated with nausea, vomiting, fitting or vertigo. Movements of all four limbs were normal. Past medical history failed to disclose any serious illness or congenital abnormalities.

\section{Clinical examination}

Patient was cardiovascularly stable (blood pressure, $110 / 70$ and pulse, 100 beats/min). A firm swelling was located at the right pre-auricular area, measuring $8 \times 5$ $\mathrm{cm}$ with a smooth surface and normal overlying skin. His mouth opening was reduced to $1 \mathrm{~cm}$. Cranial nerve examination revealed right abducens nerve palsy and anaesthesia in the distribution of the mandibular branch of the right trigeminal nerve. Peripheral nerve examination was otherwise normal.

\section{Investigation}

Computed tomograpy (CT) scan examination revealed a large mass located primarily in the right infratemporal fossa with medial extension causing erosion of the base of skull, involvement of the right cavernous sinus. This mass encroached upon the abducent nerve, 
dura and meninges of the right temporal lobe and caused pressure resorption at the right sigmoid notch area of the ramus of mandible (Figure 2).

\section{Diagnosis}

A CT scan guided needle biopsy was done and the mass was diagnosed as rhabdomyosarcoma. Total surgical excision was not possible due to the size and intracranial extension of the lesion. Further treatment was aimed at reducing tumour size by a combination of multidrug chemotherapy and radiotherapy. There was a positive response to treatment with partial return of sensation at one year postradiotherapy.

\section{Case 3}

\section{History}

A 42-year old Chinese lady was referred to the Department of Oral \& Maxillofacial Surgery by the Oral Medicine clinic with severe unremitting pain and numbness in the right mental region of one-week duration. The patient was originally referred to the Oral Medicine clinic by her GDP because of unresolved episodes of severe pain in the right mandible of three months duration associated with a diffuse swelling of the mandible. The pain was precipitated by an extraction of a wisdom tooth and was felt over the right side of the face including the temporomandibular joint. Over the past three months, she claimed that the mandibular pain sometimes occurred in conjunction with a similar pain felt in her legs. She had no other neurological problems.

Her past medical history was significant for breast cancer. The patient was previously diagnosed with stage 4 breast adenocarcinoma ten months earlier. Her treatment plan had included several courses of radiotherapy and chemotherapy with Tamoxifen. When questioned further about follow-up, she reluctantly admitted to defaulting chemotherapy.

\section{Clinical examination}

Extraoral examination revealed a symmetrical face. Cranial nerves functions were intact except in the area of the mental nerve. The temporomandibular joint showed normal range of movements. Intraorally, the site of complaint was the right retromolar-ascending ramus region. The overlying mucosa was normal where the extraction site had healed completely. The bony contour was normal but it was tender to palpation. The tongue movement and sensation was normal.

\section{Investigations}

An OPG revealed a well-defined, unilocular radiolucency involving the ascending ramus from just below the sigmoid notch to the molar region of the body of mandible (Figure 3). Occlusal film showed a lack of bony expansion. A provisional diagnosis of metastatic cancer was made. CT scan showed multiple small lesions in the lung and liver. Total body bone-scan revealed photolucent areas in the lower thoracic and lumbar spine, right femur, both iliac crests and roof of acetabular fossa indicating widespread bony metastases.

\section{Diagnosis}

An incisional biopsy was carried out and subsequent histopathological examination confirmed the provisional diagnosis of metastatic tumour from the breast to the mandible.

\section{DISCUSSION}

The discussion is subdivided into two main portions; (a) Causes and mechanism of mental nerve paraesthesia and (b) Clinical approach to patients presenting with a numb chin.

\section{Causes and Mechanisms of Mental Paraesthesia}

In the three cases illustrated in this paper, mental nerve neuropathy were caused by two different pathological processes, malignancy and infection.

\section{Infection}

Mental paraesthesia has been described as one of the classical presentations of subacute osteomyelitis (1).

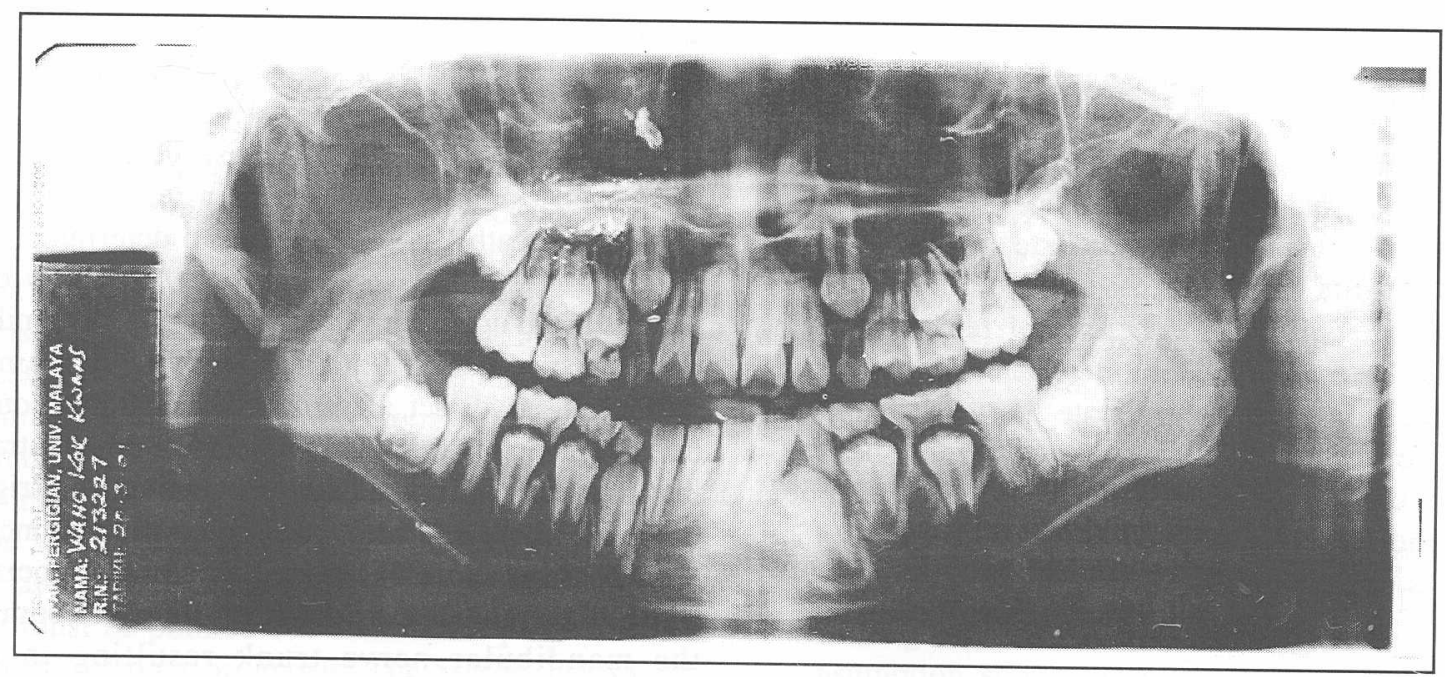

Figure 2: Orthopantomogram of patient in case 2. Widening of right sigmoid notch with an ill-defined radiolucencies in the upper half of right mandibular ramus. 


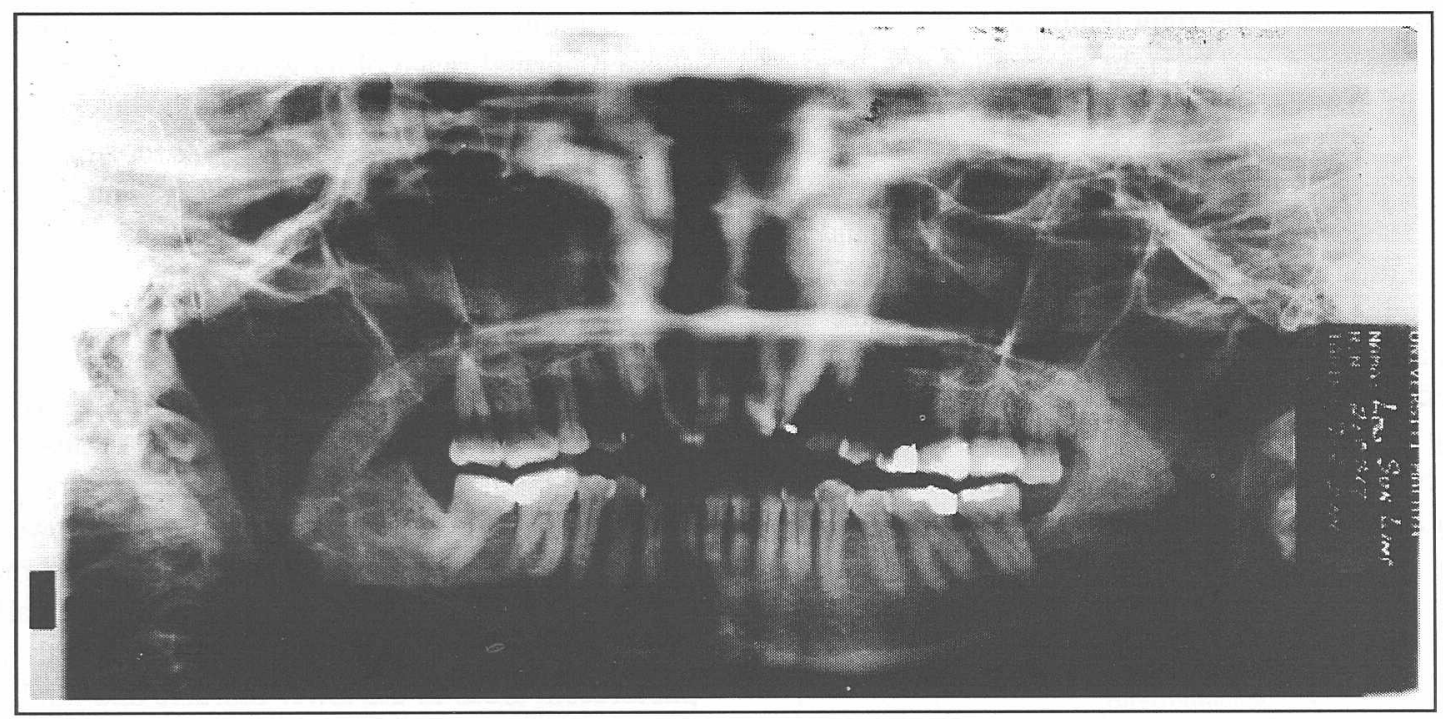

Figure 3: Orthopantomogram of patient in case 3. Radiolucent lesion in right ramus of mandible with irregular margin.

This paraesthesia may be brought about by several bacterial-related factors that could act singly or in combination. These include direct nerve compression due to a localised collection of pus, nerve damage from bacterial toxins and indirectly by disturbing the blood supply to the nerve. Occlusion of the arterioles responsible for supplying the nerve (vasa nervosa) may occur through the action of bacterial enzymes such as coagulase.

Super-infection of a chronic, non-healing mandible with compromised blood supply by bacteria is not uncommon, particularly following tooth extraction. The tooth socket failed to heal and the development of subacute osteomyelitis is encouraged by poor local blood supply. This occurrence has been documented in a previous report of poor socket healing associated with osteomyelitis in florid cemento-osseous dysplasia (2).

Florid cemento-osseous dysplasia (FCOD) is a lesion forming part of the fibro-osseus dysplasia group. It has previously been referred to under several terms: gigantiform cementoma, sclerosing osteitis, multiple enostosis and diffuse sclerosing osteitis due to its characteristic radiographic appearance (3). These lesions are described as multiple, areas of increased radiodensity occurring bilaterally and often symmetrical, which may frequently be associated with the apices of teeth (4).

The development of subacute osteomyelitis related to FCOD results from the presence of these dense, calcified tissues around the periapical region of teeth intended for extraction. This often demands excessive use of force in attempt to extricate the tooth from the jaw. Frequently these bulky masses are left behind, forming a cuff of physical barrier around the tooth socket, hence severely restricting its blood supply that predisposes to osteomyelitis.

\section{Malignancy}

The presentation of mental nerve neuropathy owing to malignancy is well documented in the literature and is often described as the 'numb chin syndrome' (5), (6). Numb chin syndrome has also been used by others to describe mental paraesthesia irrespective of its causes $(7,8,9)$.

The use of this term under those circumstances may seem inappropriate as the term 'syndrome' is categorically used to describe a combination of signs and/or symptoms that forms a distinct clinical picture indicative of a particular disorder. In doing so too, the significant clinical impact of a numb chin ascribed to malignancy becomes somewhat diluted when numb chin syndrome is used to describe benign causes of mental paraesthesia. Therefore, it is suggested that the term 'numb chin syndrome' should be used specifically in cases of mental paraesthesia attributed to malignancy only.

The most prevalent types of cancers associated with numb chin syndrome are nasopharyngeal carcinoma, meningioma, neurofibroma of the trigeminal nerve and other tumours located near the base of skull or within the mandible (7). Numb chin has also been reported as manifestations of haematological malignancies (10).

Mental neuropathy associated with malignancies may occur as the initial presentation or with systemic dissemination of the basic disease. Therefore, when a malignant lesion is encountered in relation to a mental nerve neuropathy, it is important to determine whether the tumour is a primary lesion or a secondary deposit. However, the underlying mechanism may be similar. The mechanisms thought to be involved in numb chin syndrome are direct infiltration or compression of the inferior alveolar or mental nerve by tumour, intracranial involvement of the mandibular branch of the trigeminal nerve by lesions at the base of skull or leptomeningeal seeding of tumour (11). In the second case reported, the primary tumour in the pterygoid fossa had compressed the mandibular nerve trunk resulting in mental anaesthesia. The tumourous pterygoid muscle also limited the mouth opening. 
Rhabdomyosarcoma (RMS) is the most common (70\%) type of primary malignant mesenchymal tumour of childhood and adolescence with 29\% of the cases occurring in the head and neck region (International Society of Paediatric Oncology). In this group, parameningeal sites (i.e. paranasal sinuses, nasopharynx, middle ear, pterygoid fossa and other sites with meningeal involvement and/or bone destruction) were more common than non-parameningeal sites (12). Diagnosis of parameningeal RMS (pRMS) is frequently made later than non-parameningeal cases because of the deeper location of the tumours, as observed in the second case.

RMS involvement of the infratemporal fossa is not common, only three such cases have been reported in the English literature $(13,14)$. Tumours in the infratemporal region are often diagnosed late, due to their deep location (15), and, are usually made obvious only after the presence of pre-auricular swellings as seen in our patient. Therefore, it was not unexpected when CT scan images showed the lesion to be at an inoperable size.

In the patient described, apart from the numb chin other aspects indicating the diagnosis, site and extent of RMS were his age, the presence of headache suggestive of intracranial involvement, limitation of mouth opening and cranial nerve palsy in the absence of other neurological deficits.

Metastatic tumours make up to about $1 \%$ of all oral malignancies (16). In a meta-analysis of metastatic tumour to the jaws, the most common primary tumour to metastasise to the jaws in women was breast carcinoma while the lung is the most frequent primary site in men $(17,18)$. In about $30 \%$ of these patients, the clinical presentation of metastatic jaw disease was the first indication of an existing malignancy.

While a numb chin is a well documented sign of metastatic lesion, other signs and symptoms suggestive of this condition include swelling, severe unremitting pain resembling toothache, facial pain, pathological fracture, limitation of mouth opening, facial and other cranial nerve palsies $(17,19,20)$. The mandible is by far more common than the maxilla as a site for distant spread. The location of these metastatic deposits were frequently found in the mandibular premolar-molar region thereby manifesting as numb chin (17).

The most important radiographic feature suggestive of metastases is an ill-defined margin surrounding lesions that could be completely radiopaque or radiolucent and sometimes mixed radiopaque-radiolucent (17). Interestingly, the radiological appearance of jaw metastases may be similar to osteomyelitis. In such instances, one of the ways to differentiate them is by using an anti-granulocyte monoclonal antibody labelled bone marrow scan in which the take-up will be seen at sites of infection (20).

The appearance of mental paraesthesia from the time of primary cancer diagnosis averaged at 39.5 months (17). It must be pointed out that mental paraesthesia is an indicator of poor prognosis. The 5-year survival rate of patients with jaw deposits is estimated at only $10 \%$ and the mean duration from the presentation of mental paraesthesia to death was found to be merely 7.3 months (17).

In the last case presented, the patient had a primary adenocarcinoma of the breast. Her past medical history provided the crucial information. Although she was diagnosed only 10 months ago, she had abandoned her treatment halfway through its course. Despite experiencing only a short duration of numbness, the most telling symptom was the repeated episodes of severe unremitting pain. The presence of neurological dysesthesia coupled with a history of breast carcinoma was highly suggestive of a metastatic disease. Subsequent clinical examination confirmed the impression collated from the history obtained.

\section{b. Clinical approach}

Numbness of the chin is an important clinical presentation because it may be caused by a significant underlying pathology. Although time may be a constraint in a busy dental practice, a complete history and systematic examination of a patient with such a problem may be worthwhile for both patient and the dentist. To ensure that important questions and signs are not missed, the possible causes of mental paraesthesia should be referred to (Figure 1).

The onset of paraesthesia provides vital clues to its aetiology. Precipitating factors such as a history of trauma to the jaws, previous surgical procedures to the mandible and complex dental treatment such as endodontics are potential culprits and must be enquired. Numbness following extraction of a tooth may suggest the presence of subacute osteomyelitis in a medically compromised patient or herald the presence of an underlying malignancy. Information regarding the course and progression of numbness sheds some light regarding the state and evolution of the underlying disease process.

It would be of extreme value to determine if the neurological dysfunction is due to a single neuron involvement (mononeuropathy) or occurs as part of a polyneuropathy. While dental-related causes tend to present as a mononeuropathy, polyneuropathy with or without other symptoms of neurological problems like headache, seizures, blackouts, weakness etc points to a more systemic aetiology. A persistent dysesthesia of nondental origin deserve a referral to the hospital-based physician for further management.

Obtaining the personal and social history is equally important in order to rule out inorganic causes such as hysterial anaesthesia of the face (1), which is now widely recognised in the medical literature as somatisation. According to Harris, by accepting functional loss from the numbness, patients gain a better footing psychologically by receiving sympathy, attention and escaping responsibility. The character of numbness that is described by these patients is haphazard and does not usually fit into the usual pattern of neurological diseases.

Indulgence in habits that predispose patients to a wide range of disease such as tobacco usage and alcohol consumption should be noted particularly the amount taken daily and length of habit. Environmental causes 
of neuropathy are very rare. However, some industrial agents known to be neurotoxic include certain organic solvents, toluene, arsenic and lead.

Eliciting and documenting the past medical history is an essential component in the understanding the cause of a numb chin. A history of diabetes mellitus should be asked directly. Peripheral as well as autonomic neuropathy is a common complication of diabetes mellitus and is related to poor diabetic control. Although the classical presentation of a diabetic neuropathy is the so-called 'glove and stocking' distribution, any nerve can be affected.

A previous diagnosis of malignancy and its treatment must be enquired. In men, the primary sites that frequently metastasize to the jaws are the lung, kidney and colo-rectal cancers, while in females the sites are breast, female genitalia and the kidneys (18). As metastatic jaw disease is the first presenting sign of a distant malignancy in about $30 \%$ of cases, it is vital to ask about the presence of site-specific symptoms rather than direct questioning. Other medical conditions that may be of relevance include vascular conditions, connective tissue disease, kidney and liver problems. It is also very useful to undertake a systems review to screen for other symptoms particularly in the situation where the patient is unable to provide a clear record of their previous medical problems.
Drugs are a common cause of nerve disturbances and it is necessary to obtain a full drug list from the patient. Only one drug, hydroxystillbamidine (antiprotozoal) has been reported in the literature as a direct cause of bilateral mental paraesthesia (1). Other potentially neurotoxic drugs include certain antibiotics (e.g. isoniazide, metronidazole), chemotherapeutic agents (e.g. vincristine, cisplatin) and some psychotherapeutic agents (e.g. lithium, tricyclic antidepressants).

Information regarding the family medical history is valuable particularly in highlighting the risk of diabetes mellitus, cardiovascular disease, thyroid disease and cancers that have a tendency to cluster in families especially breast and colorectal cancer. Furthermore, in young patients, the possibility of an inheritable disease as a cause of their problem is likely.

Clinical examination of a patient with a numb chin should ideally involve a complete physical examination and this may not be appropriate in the setting of a dental practise. However, much information can be drawn just from an adequate history and keen observation of the patient (Figure 4). As the patient enters the surgery, it is possible to assess the patient's general health, gait, balance and coordination. Observing the patient's response to your questions during history taking will give clues to the patient's intellectual skills, speech, articulation, attention, orientation and mood.

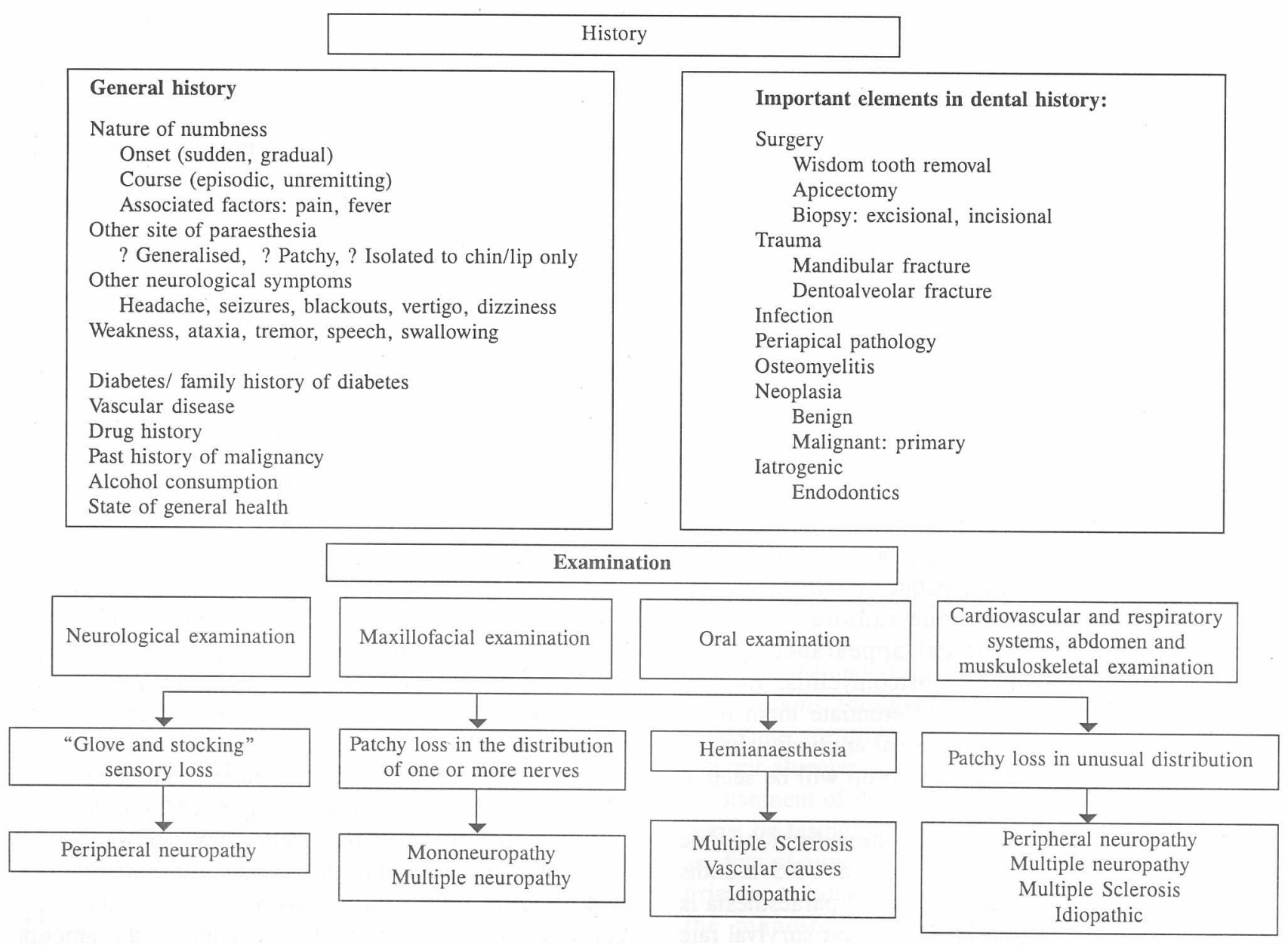

Figure 4: Flow chart in clinical approach to mental nerve paraesthesia. 


\begin{tabular}{|l|}
\hline Higher cerebral function \\
\hline 1. State of \\
consciousness \\
Alert / Confused \\
2. Intellectual skills \\
Orientation \\
Memory \\
Concentration \\
General knowledge \\
\\
3. Mood/ Emotions \\
Anxious/ Depressed \\
4. Speech \\
Problems with: \\
Articulating words \\
Understanding \\
speech \\
Naming objects \\
\hline
\end{tabular}

\begin{tabular}{|l|}
\hline \multicolumn{1}{|c|}{ Cranial nerves } \\
\hline 1. Olfactory nerve \\
Ask about sense of smell \pm taste \\
2. Optic nerve \\
Visual acuity (Finger count) \\
Visual fields \& Light reflex \\
3. Occulomotor, Trochlear \& \\
Abducen \\
Eye movements \\
4. Trigeminal nerve \\
Sensory : Corneal reflex \\
$\quad$ Refer to 'sensation' \\
Muscles of mastication \\
$\quad$ Palpate masseter, temporalis \\
5. Facial nerve \\
Ask patient to: raise eyebrows, \\
screw eyelids shut, give a toothy \\
smile, blow cheeks out \\
Taste of anterior $2 / 3$ rd tongue \\
Hyperacusis \\
6. Vestibulococchlear nerve \\
Vertigo \\
Assess hearing: rub finger tips, \\
whisper in each ear \\
7. Glossopharyngeal \& Vagus \\
nerves \\
Say 'aahh' : ? palatal palsy \\
Gag reflex \\
8. Accessory nerve \\
Shrug shoulders and turn head \\
laterally against resistance \\
9. Hypoglossal nerve \\
Stick tongue out.: ?deviated, \\
?fasciculation \\
\hline
\end{tabular}

\begin{tabular}{|l|}
\hline \multicolumn{1}{|c|}{ Motor system and Gait } \\
\hline Inspection: \\
Wasting, Fasciculation, Deformity, \\
Scar \\
Palpation: \\
Tone: means how floppy ( $\downarrow$ tone) \\
or stiff ( $\uparrow$ tone) limb feels \\
Power: Compare with opposite side \\
relative to examiner's power \\
Reflexes: Grade as increased, \\
decreased or absent \\
1. Upper limb \\
Tone: Flex and extend arm \\
Power: 'Squeeze my fingers' \\
Coordination: 'Finger to nose test' \\
Reflexes: Biceps, Triceps, \\
Suppinator \\
2. Lower limb \\
Tone: 'Lift legs up' (while on \\
dental chair) \\
Power: 'Lift legs up' against \\
resistance; \\
'Kick out' and 'Bend knee' against \\
resistance; Ankle dorsi- and \\
plantar-flexion against resistance \\
Reflexes: Knee, Ankle and \\
Babinski \\
Gait \\
Look as patient walks into surgery OR \\
ask patient to walk up and down the \\
surgery. \\
Observe: abnormalities of posture, \\
length of feet separation, arm and leg \\
swing, control of upper body. \\
\\
\end{tabular}

\begin{tabular}{|l|}
\hline \multicolumn{1}{|c|}{ Sensation } \\
\hline Keep it simple by tailoring \\
examination to patient's \\
complaint. \\
Explain to patient what is \\
being tested so patient can \\
feel certain sensations. \\
Test from abnormal area \\
outwards \\
1. Pin prick \\
Testing for 'pain' \\
sensation. \\
Use edge of paper clip, \\
(not needle) patient's \\
eyes open and ask \\
'sharp or blunt?' \\
2. Light touch \\
Use cotton wool and \\
patient's eyes closed. \\
Can the patient feel \\
cotton wool touching \\
skin? \\
3. Two-point \\
discrimination \\
Use the two edges of \\
paper clip at a fixed \\
distance ( 2 and 5 mm) \\
with patient's eyes \\
closed. \\
Can the patient feel one \\
or two points? \\
4. Temperature \\
Eyes closed. \\
Use lukewarm object \\
for hot and ethyl \\
chloride for cold. \\
Ask patient if feels cold \\
or hot? \\
\hline
\end{tabular}

Figure 5: Simple method of neurological examination in a dental surgery setting.

The cranial nerves and peripheral nerves may be assessed by the method suggested in Figure 5. The chart is a simplified version of the normal sequence of neurological examination and a more detailed explanation is available in most textbooks of medicine. While examining, it is important to consider the anatomical site of lesion and the possible underlying pathological process. From the perspectives of a numb chin, the neurological findings may be considered into four main patterns. At this point, the dentist should be able to decide if the patient's problem may be managed at the surgery or requires prompt referral to the hospital.

Although many of the important investigations for a numb chin may need to be carried out in a hospital, the most useful investigation that may be carried out in a dental surgery is the dental radiograph. Of all dental radiographs, the ortopantomogram is the most important as this film provides an overall view of the maxillofacial region.

\section{CONCLUSIONS}

Numbness of the chin is a common occurrence in the dental practice. It should be a cause for concern as it may indicate a serious underlying pathology, such as malignancy. Numb chin associated with malignancy should be referred to as the 'numb chin syndrome'. The quality and distribution of the symptoms may suggest the site of the lesion and nature of the disease. The dental surgeons should try to determine from the history and clinical examination whether the mental paraesthesia is likely to result from diseases requiring hospital-based management. This paper outlines the important aspects of history taking and examination of a patient presenting with a numb chin based on the list of its differential diagnosis. 


\section{REFERENCES}

1) Harris M. Disturbance of facial sensation. Oral Surg Oral Med Oral Pathol 1967; 24: 335-347.

2) Gingrass DJ, Sadeghi EM, Eslami A. Florid osseous dysplasia: clinical, histopathologic and therapeutic considerations. Compendium 1986; 7: 731-736.

3) Waldron CA. Fibro-osseous lesions of the jaws. J Oral Maxillofac Surg 1985; 43: 249-262.

4) Melrose RJ, Abrams AM, Mills BG. Florid osseous dysplasia. A clinico-pathologic study of thirty-four cases. Oral Surg Oral Med Oral Pathol 1976; 41(1): 62-82.

5) Halachmi S, Madeb R, Madjar S et al. Numb chin syndrome as a presenting symptom of metastatic prostate carcinoma. Urology 2000; 55: 286.

6) Lossos A, Siegal T. Numb chin syndrome in cancer patients: etiology, response to treatment and prognostic significance. Neurology 1992; 42: 11811184.

7) Sebor RJ. Numb chin syndrome: a case report. Compendium 1990; 11(10): 620,622,624-625.

8) Thomson PJ, Rood JP. Mental nerve dysfunction: A symptom of diverse mandibular disease. Dental Update 1995; September: 271-274.

9) Friedlander $\mathrm{AH}$, Monson $\mathrm{M}$. Other causes of the numb chin. J Oral Maxillofac Surg 1993; 51(2): 217.

10) Hiraki A et al. Numb chin syndrome as an initial symptom of acute lymphocytic leukemia. Oral Surg Oral Med Oral Pathol Oral Radiol Endod 1997; 83: 555-61.

11) Girolami U, Anthony DC, Frosch MP. Peripheral nerve and skeletal muscle. Robbins pathologic basis of disease. $6^{\text {th }}$ edn. Chicago, W.B. Saunders Company 1999; pp.1275-1280.
12) Flamant F, Habrand J, Lacombe MJ, Revillon Y. Malignant mesenchymal tumours in childhood. In Peckham, Pinedo \& Veronesi (Eds). Oxford Textbook of Oncology. $3^{\text {rd }}$ edn. Oxford University Press 1995; pp. 1943-1950.

13) Dohar JE, Marentette LJ, Adams GL. Rhabdomyosarcoma of the infratemporal fossa: diagnostic dilemma and surgical management. Am J Otolaryngol 1991; 12: 146-149.

14) Goto TK, Yoshiura K, Tanaka T et al. A follow-up of rhabdomyosarcoma of the infratemporal fossa region in adults based on magnetic resonance imaging: case reports. Oral Surg Oral Med Oral Pathol Oral Radiol Endod 1998; 86: 616-625.

15) Johnson AT, Maran AGD. Extracranial tumours of the infratemporal fossa. J Laryngol Otol 1982; 96: 1017-1026

16) Meyer I, Shaklar G. Malignant tumours metastatic to mouth and jaws. Oral Surg 1965; 20: 350-362.

17) Hirshberg A, Leibovich P, Buchner A. Metastatic tumours to the jaw bones: analysis of 390 cases. J Oral Pathol Med 1994; 23: 337-341.

18) Hirshberg A, Buchner A. Metastatic tumours to the oral region. An overview. Eur J Cancer Oral Oncol 1995; 31B(6): 355-360.

19) Pruckmayer M, Glaser C, Marosi C, Leitha T. Mandibular pain as a leading clinical symptom for metastatic disease: nine cases and review of the literature. Ann Oncol 1998; 9: 559-564.

20) Glaser C, Lang S, Pruckmayer $M$ et al. Clinical manifestation and diagnostic approach to metastatic cancer of the mandible. Int J Oral Maxillofac Surg 1997; 26: 365-368. 